\title{
Decay of Benjamin-Ono solitons under the influence of dissipation
}

\author{
Roger H. Grimshaw ${ }^{a)}$, Noel F. Smyth ${ }^{b)}$, Yury A. Stepanyants ${ }^{c, d)} 1$ \\ a) University College London, UK; \\ b) School of Mathematics, University of Edinburgh, \\ James Clerk Maxwell Building, The King's Buildings, \\ Peter Guthrie Tait Road, Edinburgh, Scotland, U.K., EH9 3FD; \\ ${ }^{c)}$ Faculty of Health, Engineering and Sciences, \\ University of Southern Queensland, Toowoomba, QLD, 4350, Australia and

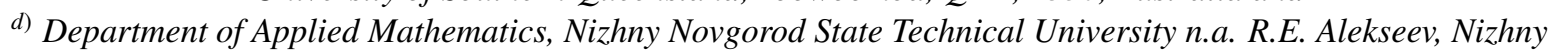 \\ Novgorod, Russia.
}

\begin{abstract}
The adiabatic decay of Benjamin-Ono algebraic solitons is studied when the influence of various types of small dissipation and radiative losses due to large scale Coriolis dispersion are taken into consideration. The physically most important dissipations are studied, Rayleigh and Reynolds dissipation, Landau damping, dissipation in a laminar boundary layer and Chezy friction on a rough bottom. The decay laws for the soliton parameters, that is amplitude, velocity and width, are found in analytical form and are compared with the results of direct numerical modelling. Keywords: internal wave, Benjamin-Ono equation, soliton, Rayleigh dissipation, Reynolds dissipation, Landau damping, Chezy friction, rotating fluid, adiabatic decay
\end{abstract}

\footnotetext{
${ }^{1}$ Corresponding author, e-mail: Yury.Stepanyants@usq.edu.au Preprint submitted to Elsevier
} 


\section{Introduction}

The propagation of small amplitude long waves in a stratified fluid consisting of a relatively thin layer overlying a very deep passive layer is described by the well-known Benjamin-Ono (BO) equation $[1,2,8]$

$$
\frac{\partial u}{\partial t}+\alpha u \frac{\partial u}{\partial x}+\frac{\beta}{\pi} \frac{\partial^{2}}{\partial x^{2}} \wp \int_{-\infty}^{+\infty} \frac{u(\xi, t)}{\xi-x} d \xi=0 .
$$

Here, $u(x, t)$ is the perturbation of a pycnocline (a layer with a constant density) and $\alpha$ and $\beta$ are parameters which depend on the particular stratification (for details see [2, 8]). The symbol $\wp$ denotes the principal value of the integral. The BO equation (1) is set in a coordinate frame moving with the speed $c$ of linear long waves. In particular, the BO equation has the algebraic soliton solution [1]

$$
u(x, t)=\frac{A}{1+(x-V t)^{2} / \Delta^{2}} .
$$

Here, $A$ is the soliton amplitude, $V=\alpha A / 4$ is its velocity in the Galilean coordinate frame moving with the speed $c$ with respect to an immovable observer and $\Delta=4 \beta / \alpha A$ is its characteristic width.

The BO equation is similar to the classic Korteweg-de Vries (KdV) equation for weakly nonlinear long waves in a shallow fluid

$$
\frac{\partial u}{\partial t}+\alpha u \frac{\partial u}{\partial x}+\beta \frac{\partial^{3} u}{\partial x^{3}}=0 .
$$

In particular, both are completely integrable [1] and have families of periodic wave solutions, one limit of which is the solitary wave solution. A specific feature of these solitary wave solutions is that they are robust and restore their parameters (amplitude, shape etc.) after collisions with each other, so that they are termed solitons [33], the only relic of the interaction being a phase shift. They are also stable under arbitrary localised perturbations.

In real physical media there are usually different dissipative mechanisms which affect wave shapes and soliton dynamics. The influence of various types of dissipation on solitons and kinks has been studied in detail based on various model evolution equations (see, for instance, [11]). However, the influence of dissipation on the decay of BO solitons has not been studied as yet. In this paper we fill this gap and study the adiabatic decay of algebraic BO solitons under the influence of weak dissipation of various types; Rayleigh and Reynolds dissipation, Landau damping, 
dissipation in a laminar boundary layer and Chezy friction when the soliton propagates over rough bottom topography, as well as the dissipation caused by the radiation of small amplitude waves in media containing weak large scale dispersion. Such dispersion typically arises in a rotating fluid [7, 10], but can be caused by specific dispersion dependences in other media.

If weak dissipation is taken into account the BO Eq. (1) is augmented by additional terms whose structure depends on the nature of this dissipation. In general, BO type equations with dissipative terms can be presented in the form

$$
\frac{\partial u}{\partial t}+\alpha u \frac{\partial u}{\partial x}+\frac{\beta}{\pi} \frac{\partial^{2}}{\partial x^{2}} \wp \int_{-\infty}^{+\infty} \frac{u(\xi, t)}{\xi-x} d \xi+\delta \mathcal{D}[u]=0,
$$

where $\mathcal{D}[u]$ is an operator which can be expressed in the rather general form [8]

$$
\mathcal{D}[u]=\frac{1}{\sqrt{2 \pi}} \int_{-\infty}^{+\infty}(-i k)^{m} \tilde{u}(k, t) e^{i k x} d k \quad \text { and } \quad \tilde{u}(k, t)=\frac{1}{\sqrt{2 \pi}} \int_{-\infty}^{+\infty} u(x, t) e^{-i k x} d x .
$$

Here $\tilde{u}(k, t)$ is the Fourier transform of the function $u(x, t)$ and the parameter $m$ depends on the specific type of dissipation.

The case $m=0$ (together with $\delta>0$ ) corresponds to linear Rayleigh damping, as the dissipative term $\mathcal{D}[u]$ in Eq. (4) reduces simply to $\delta u$. This loss has been invoked in the internal wave context as a model for friction in the bottom boundary layer [11]. Another widely used model in many physical contexts has $m=2$ and $\delta<0$. This corresponds to Reynolds dissipation, $\mathcal{D}[u]=-\delta u_{x x}$, and so $\delta$ represents the kinematic viscosity of the fluid.

When $m$ is not an even number the term $(-i k)^{m}$ needs a more careful interpretation. In particular, when $m=1$ it should be replaced by $|k|$. In this case the dissipative term $\mathcal{D}[u]$ reduces to the Hilbert transform of the derivative $u_{x}$ and describes the Landau damping of plasma waves, or of internal waves in a stratified fluid with a shear flow [25, 26] (see Eq. (26) below for an alternative representation of the $\mathrm{BO}$ equation with Landau damping).

For non-integer $m$ the correct interpretation of the term $(-i k)^{m}$ is

$$
(-i k)^{m}=|k|^{m} \exp (-i \operatorname{sign}[k] m \pi / 2),
$$

where sign $[k] \equiv k /|k|$. This representation ensures that $\mathcal{D}[u]$ is real valued when $u$ is real valued, as it can be readily verified in this case that $\mathcal{D}[u]^{*}=\mathcal{D}[u]$, noting that $\tilde{u}^{*}(k)=\tilde{u}(-k)$ (here the star 
superscript denotes the complex conjugate). When $m=1 / 2$ this dissipative term can be used for the description of wave decay due to a laminar bottom boundary layer [8].

The operator $\mathcal{D}[u]$ can be nonlinear. An important example is $\mathcal{D}[u]=|u| u$ for the dissipation of internal solitary waves over a rough bottom [8]. This model is based on the empirical description of dissipation in a turbulent boundary layer.

Note that for $m>0$ solutions of the BO Eq. 4 . conserve "mass" $M=\int_{-\infty}^{\infty} u(x, t) d x$, whereas for $m=0$ the total mass is not conserved. This issue has been discussed by Miles [20] in the application of the $\mathrm{KdV}$ equation to water waves in a channel which has variable depth and width. In particular, in the case of Rayleigh dissipation, on integrating the perturbed BO Eq. (4) we obtain the mass balance equation

$$
\frac{d M}{d t}=-\delta M
$$

which has the solution $M(t)=M_{0} e^{-\delta t}$. It is interesting to note that for the BO soliton (2) the total mass $M_{s}=4 \pi \beta / \alpha$ does not depend on its amplitude. It is then concluded that the mass balance (7) implies that under the influence of even small Rayleigh dissipation the solution $u(x, t)$ cannot be just a BO soliton, but must contain a non-solitonic part (a trailing wave).

A shelf forms behind the evolving soliton under the influence of Chezy friction for a similar reason based on mass balance. In this case, the mass balance equation gives

$$
\frac{d M}{d t}=-\delta \int_{-\infty}^{+\infty}|u| u d x .
$$

If the initial condition is a $\mathrm{BO}$ soliton, then the mass decay rate at $t=0$ is $-2 \pi \beta \delta / \alpha$, which is two times less than in the case of Rayleigh decay. The complete solution for $M(t)$ then requires a knowledge of the solution for $u(x, t)$, including the non-solitonic contribution behind the soliton.

When the dissipation is sufficiently small, one would expect that the basic structure of the solitary wave remains the same, but its primary parameter $A$, which determines its amplitude, speed and width, is no longer a constant, but is a slowly varying function of time. This dependence on time can be calculated by means of asymptotic theory [5, 8, 24] which presumes that the soliton adiabatically varies with time, while retaining its shape. This asymptotic theory in essence reduces to an energy balance equation which describes the time dependence of the governing 
soliton parameter $A$. In terms of a multiple scales analysis, this energy equation is the condition for the elimination of secular terms. Multiplying Eq. (4) by $u$ and then integrating over $x$, we obtain the energy balance equation

$$
\frac{d E}{d t}=-\delta F
$$

where the "wave energy" $E$ and dissipative function $F$ are

$$
E=\frac{1}{2} \int_{-\infty}^{+\infty} u^{2}(x, t) d x, \quad F=\int_{-\infty}^{+\infty} u(x, t) \mathcal{D}[u] d x .
$$

Using Parseval's theorem [4], we can calculate the wave energy as

$$
E=\frac{1}{4 \pi} \int_{-\infty}^{+\infty}|\hat{u}|^{2} d k
$$

The convention (6) for $(-i k)^{m}$ ensures that $F$ is real valued. It can then be presented in the alternative form as

$$
F=\frac{\sigma}{2 \pi} \int_{-\infty}^{+\infty}(-i k)^{m}|\tilde{u}|^{2} d k=\frac{\sigma}{\pi} \cos \frac{m \pi}{2} \int_{0}^{+\infty}|k|^{m}|\hat{u}|^{2} d k,
$$

where $\sigma= \pm 1$. The sign $\sigma$ should be chosen so that the dissipative function $F>0$. In particular, for $0 \leq m<1, \sigma=1$, whereas for $1<m \leq 2, \sigma=-1$. For $m=1$, that is Landau damping, expression (12) is invalid (formally it gives $F=0$ ). In this case the correct expression is

$$
F=\frac{1}{\pi} \int_{0}^{+\infty}|k||\tilde{u}|^{2} d k
$$

The wave energy $E$ is obviously conserved when there is no dissipation $(\delta=0)$. For the BO soliton (2) the energy is

$$
E_{s}=\frac{\pi}{4} \Delta A^{2}=\frac{\pi \beta A}{\alpha}
$$

and the soliton Fourier spectrum is $\tilde{u}(x)=\pi A \Delta e^{-k / \Delta}=(4 \pi \beta / \alpha) e^{-\alpha A k / 4 \beta}$.

Below we apply this asymptotic approach to calculate adiabatic soliton decay under the action of the different decay mechanisms discussed here. We then study the non-adiabatic stage of soliton decay, which occurs on very long time scales. 


\section{Rayleigh dissipation}

We first consider the effect of weak Rayleigh dissipation on the dynamics of a BO soliton. Bearing in mind that in the case of Rayleigh dissipation $\mathcal{D}[u]=\delta u$ (see above), we obtain from the energy balance equation (9)

$$
\frac{d E_{s}}{d t}=-2 \delta E_{s}, \quad E_{s}(t)=E_{0} e^{-t / \tau}, \quad A(t)=A_{0} e^{-t / \tau},
$$

where $E_{0}$ and $A_{0}$ are the initial soliton energy and amplitude, respectively (note that $E_{s} \sim A$ as per Eq. (14)). $\tau=1 / 2 \delta$ is the characteristic time of soliton decay (the time taken for the soliton amplitude to decrease by the factor $e$ ). Note that the linear wave solution of the linearised BO equation with $\alpha=0$ decays two times slower, with $\tau_{\sin }=1 / \delta$. This discrepancy in the decay rate is explained by the relationship between the soliton amplitude and width which is absent for a linear wave. Using the relationship between the soliton amplitude, speed and width (see after Eq. (2)), we find that the velocity also decreases exponentially in time with the same decay rate, whereas the width exponentially increases with time, $\Delta=\Delta_{0} e^{t / \tau}$.

To validate this asymptotic result we undertook direct numerical simulations of soliton evolution within the framework of the perturbed BO equation (4) with an additional dissipative term. The dependence of $A(t)$ for Rayleigh dissipation is shown in Fig. 1 for two values of $\delta=10^{-3}$ (dots) and $\delta=10^{-4}$ (rhombuses). As can be seen, the smaller the $\delta$, the better the agreement between the numerical data and the asymptotic theory (red line 1).

The range of validity of the asymptotic theory can be estimated on the basis of different approaches. A simple estimate can be obtained by a comparison of the relative strengths of the dissipative term $(\sim \delta A(t))$ and the nonlinear $\left(\sim \alpha A^{2}(t) / 2 \Delta(t)\right)$ or dispersive $\left(\sim \beta A(t) / \Delta^{2}(t)\right)$ terms (it is worth recalling that there is a balance of nonlinear and dispersive effects for a soliton solution, therefore any of these latter terms can be taken for the estimate). In particular, the ratio of dissipative to nonlinear terms is $8 \delta \beta / \alpha^{2} A^{2}(t)=8 \delta \beta e^{4 \delta t} / \alpha^{2} A_{0}^{2}$. This shows that the relative role of the dissipative term quickly increases. The adiabatic theory then becomes invalid when this ratio becomes of order of unity. We then obtain that the asymptotic theory is valid until

$$
t / \tau \sim \ln \frac{\alpha A_{0}}{2 \sqrt{\beta \delta}}
$$




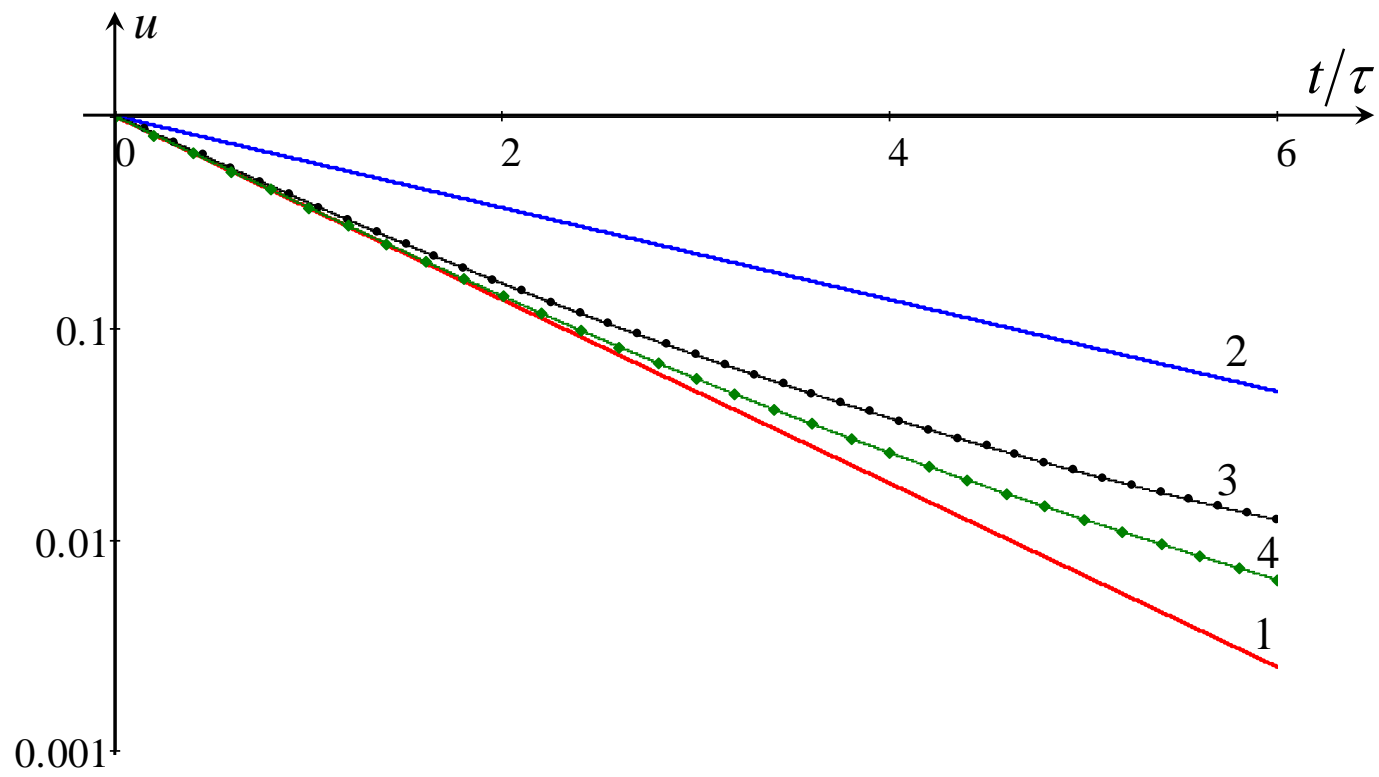

Figure 1: (colour online). The dependence of soliton amplitude on time (semi-log scale) in the case of Rayleigh dissipation. Red line 1 - the theoretical dependence; blue line 2 - the amplitude decay of a linear wave; line 3 with dots - numerical data obtained with $\delta=10^{-3}$; line 4 with rhombuses — numerical data obtained with $\delta=10^{-4}$. The other parameters are $\alpha=2$ and $\beta=1$.

Using the parameters for Fig. 1, we obtain $t / \tau \approx 3.1$ for $\delta=10^{-3}$ and $t / \tau \approx 4.3$ for $\delta=10^{-4}$, which is in agreement with the results shown in the Figure.

Another estimate for the range of validity of the asymptotic theory can be obtained from the mass balance equation (7). Assuming that in the course of the wave evolution a shelf $u_{\text {shelf }}$ is generated behind the leading solitary wave, the total wave mass is

$$
M=\int_{-\infty}^{P} u_{\text {shelf }} d x+M_{s} .
$$

Here $P(t)$ is solitary wave position, such that $P_{t}=V(t)=V_{0} e^{-2 \delta t}$. Differentiating $M$ with respect to $t$ and using the mass balance equation (7), we obtain

$$
V(t) u_{\text {shelf }}+\frac{\partial M_{s}}{\partial t}+\delta\left(M_{s}+M_{\text {shelf }}\right)=0
$$

Now the soliton mass $M_{s}=4 \pi \beta / \alpha$ is independent of time (see above). Therefore, neglecting 
$M_{\text {shelf }}$ in comparison with $M_{s}$, we obtain to first order in the parameter $\delta$

$$
u_{\text {shelf }}=-\frac{\delta M_{s}}{V(t)}
$$

Substituting the expressions for the soliton mass $M_{s}$ and speed $V(t)$, we obtain

$$
u_{\text {shelf }}=-16 \pi \delta \beta e^{2 \delta t} / \alpha^{2} A_{0}
$$

The adiabatic theory breaks down when $u_{s} \sim u_{\text {shelf }}$, which then yields

$$
\frac{16 \pi \beta \delta}{A_{0} \alpha^{2}} e^{2 \delta t} \sim A_{0} e^{-2 \delta t}
$$

This can happen quite quickly, especially for a large initial amplitude $A_{0}$, and the adiabatic theory breaks down when $t / \tau \sim \log \left(\alpha A_{0} / 4 \sqrt{\pi \beta \delta}\right)$. This estimate is similar to that derived above, Eq. (16), up to a numerical constant. However, it agrees a bit better with the numerical data shown in Fig. 1. In particular, it gives that the asymptotic theory breaks down when $t / \tau \approx 2.2$ for $\delta=10^{-3}$ and $t / \tau \approx 3.3$ for $\delta=10^{-4}$.

These estimates explain the deviation of the numerical data from the theoretical line after a certain time, as seen in Fig. 1. As has been noted by Miles [20], the results obtained within the framework of asymptotic theory when the total "wave mass" $M \neq 0$ "must be regarded with some caution. It appears likely that such solutions cannot be uniformly valid as $t \rightarrow \infty$, but this does not exclude the possibility that they are viable approximations in some contexts."

When a shelf forms behind the soliton it obeys long wave dynamics initially, so that it is governed by the approximate equation

$$
\frac{\partial u_{\text {shelf }}}{\partial t}+\delta u_{\text {shelf }} \approx 0, \quad \text { and } \quad u_{\text {shelf }} \approx u_{\text {shelf }}(x=P) \exp \{-\delta[t-R(x)]\},
$$

where $x=P(t)$ defines $t=R(x)$. Thus the shelf, which lies in $x<P$, decays exponentially behind the soliton.

Due to the influence of dissipation, the profile of the leading pulse deviates from a soliton profile. Figure 2 illustrates this phenomenon for the particular instants of time $t=10,000$ (frame a) and $t=20,000$ (frame b). As can be seen, the rear part of the leading pulse significantly differs from the soliton profile and even has an opposite polarity (see lines 2). At $t=20,000$ even 

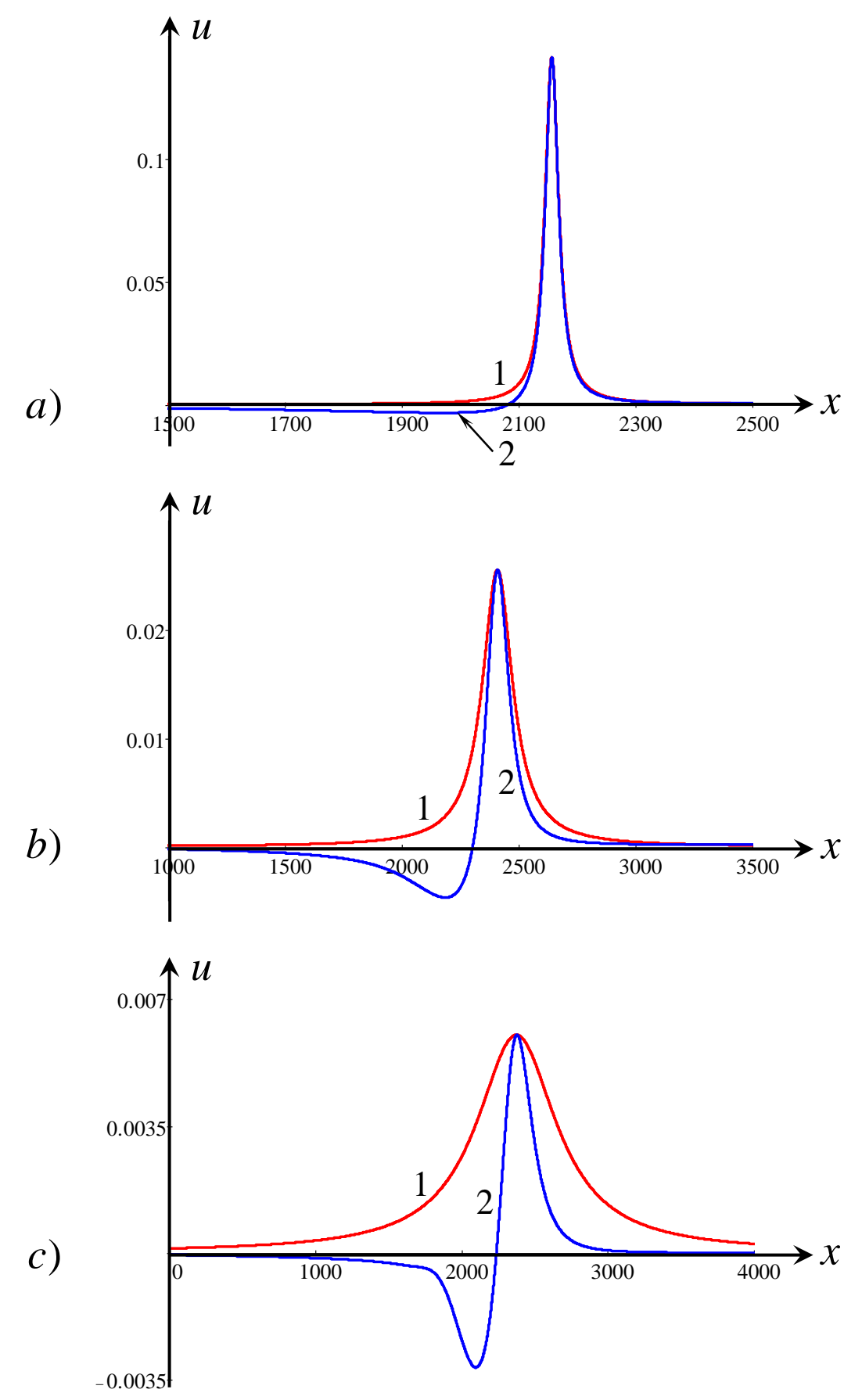

Figure 2: (colour online). Soliton decay due to Rayleigh dissipation. Solitary wave profiles (lines 2) at different instants of time compared with the BO soliton of the same amplitudes (lines 1): a) $t=10,000(t / \tau=2)$; b) $t=20,000$ $(t / \tau=4) ;$ c) $t=30,000(t / \tau=6)$. Here $\alpha=2, \beta=1$ and $\delta=10^{-4}$. 
the frontal part differs from the soliton profile. The asymptotic formula for the soliton amplitude decay (15) assumes that energy is conserved by the soliton. However, it must lose a small amount of energy due to dissipation. This could be accounted for in the higher orders of the asymptotic expansion. For large time, the tail generated behind the soliton gradually accumulates a nonnegligible amount of energy (see Fig. 2), which is not accounted for in the first order asymptotic theory. After this time, expression (15) for the soliton decay ceases to be valid.

\section{Reynolds dissipation}

We shall now consider the effect of weak Reynolds dissipation on the dynamics of a BO soliton. The BO equation with Reynolds dissipation is Eq. (4) with the dissipative term $\mathcal{D}[u]=-\delta u_{x x}$ instead of $\delta u$, where the indices of $u$ represent derivatives with respect to $x$. The energy balance equation (9) then gives

$$
\frac{d E}{d t}=-\delta \int_{-\infty}^{+\infty}\left(\frac{\partial u}{\partial x}\right)^{2} d x .
$$

The wave energy $E$ now decays non-exponentially. Substituting the soliton solution (2) into the energy evolution Eq. (23) and integrating, we obtain the soliton amplitude evolution

$$
\frac{d A}{d t}=-\frac{\delta \alpha^{2}}{16 \beta^{2}} A^{3}
$$

After integration of this equation we obtain

$$
A(t)=\frac{A_{0}}{\sqrt{1+t / \tau}}, \quad \tau=\frac{8 \beta^{2}}{\delta \alpha^{2} A_{0}^{2}}=\frac{\Delta_{0}^{2}}{2 \delta} .
$$

The theoretical dependence of the amplitude $A(t)$ is depicted in Fig. 3, together with the numerical solution. Note that for this type of dissipation the agreement between the numerical data and adiabatic theory is very good, even for a relatively large dissipation coefficient, $\delta=0.1$. This can again be explained with the help of simple estimates of the relative strength of the dissipative term $\left(\sim \delta A(t) / \Delta^{2}(t)\right)$ in comparison with the nonlinear or dispersive terms (see Sect. 2). The ratio on the dissipative term with respect to the nonlinear term is $\delta / 2 \beta$; it does not depend on time and remains small if it is initially small. It is also worth recalling that for Reynolds dissipation the total wave mass is conserved, based on the perturbed BO Eq. (4). 


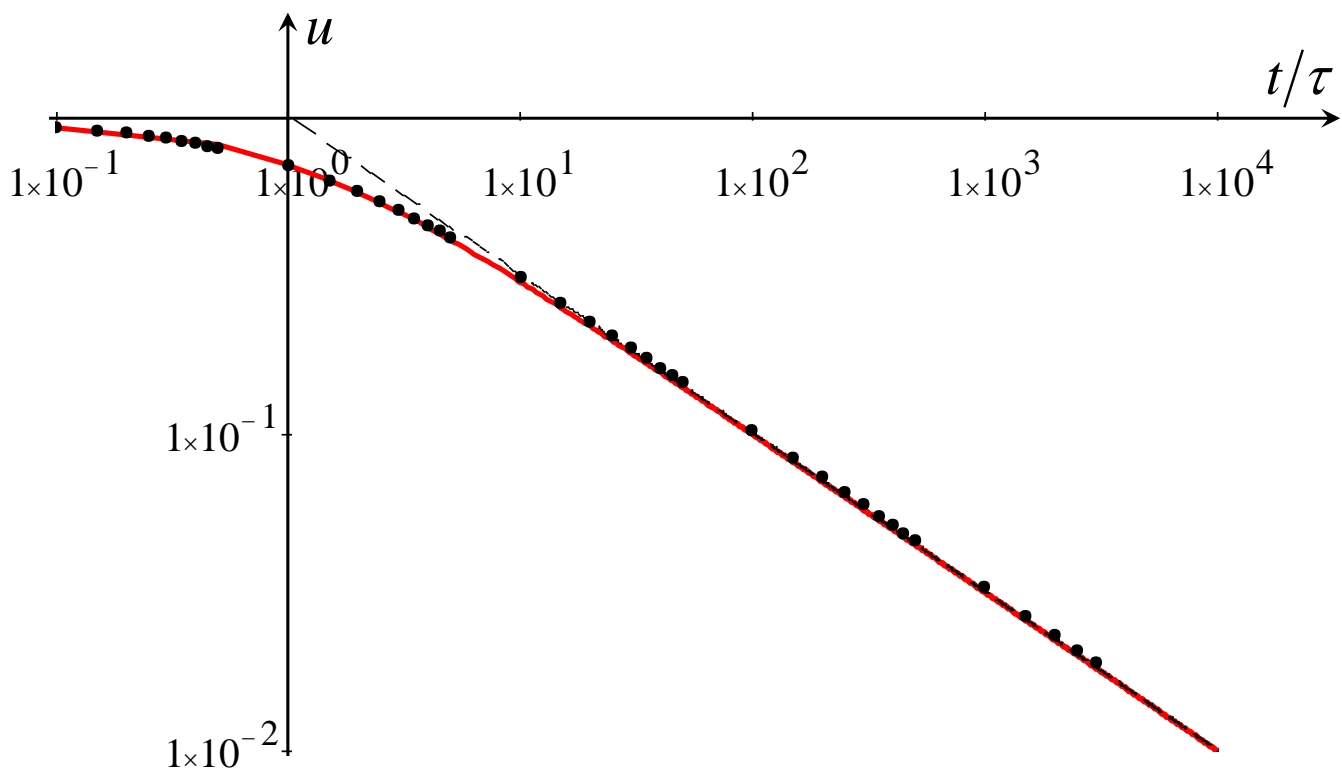

Figure 3: (colour online). Soliton amplitude dependence (log-log scale) on the normalised time $t / \tau$ for Reynolds dissipation. Solid line — theoretical dependence with $\delta=0.1$; dots — numerical data for $\alpha=2$ and $\beta=1$; dashed line - asymptotic dependence $u \sim(t / \tau)^{-1 / 2}$.

A linear wave decays exponentially due to Reynolds dissipation, $u_{\text {lin }} \sim \exp \left(-\delta k^{2} t\right)$, where $k$ is the wavenumber. Figure 4 illustrates the solitary wave profile at $t=60,000$ (line 1). At this large time the wave profile deviates slightly from a BO soliton of the same amplitude (line 1). One can clearly see the formation of a shelf behind the leading pulse in the near field, whereas in the far field the numerical solution is a decaying periodic wave.

\section{Landau damping}

This type of soliton decay applies particularly to nonlinear waves in plasma [26]. The decay of plane solitons within the framework of the $\mathrm{KdV}$ equation augmented by a dissipative term describing Landau damping has been studied as early as 1969 [26]. In the case of the BO equation (4), Landau damping corresponds to the integro-differential operator $\mathcal{D}[u]$ in Eq. (5) with the index $m=1$ in the spectral operator. The corresponding BO equation can be presented in explicit form 


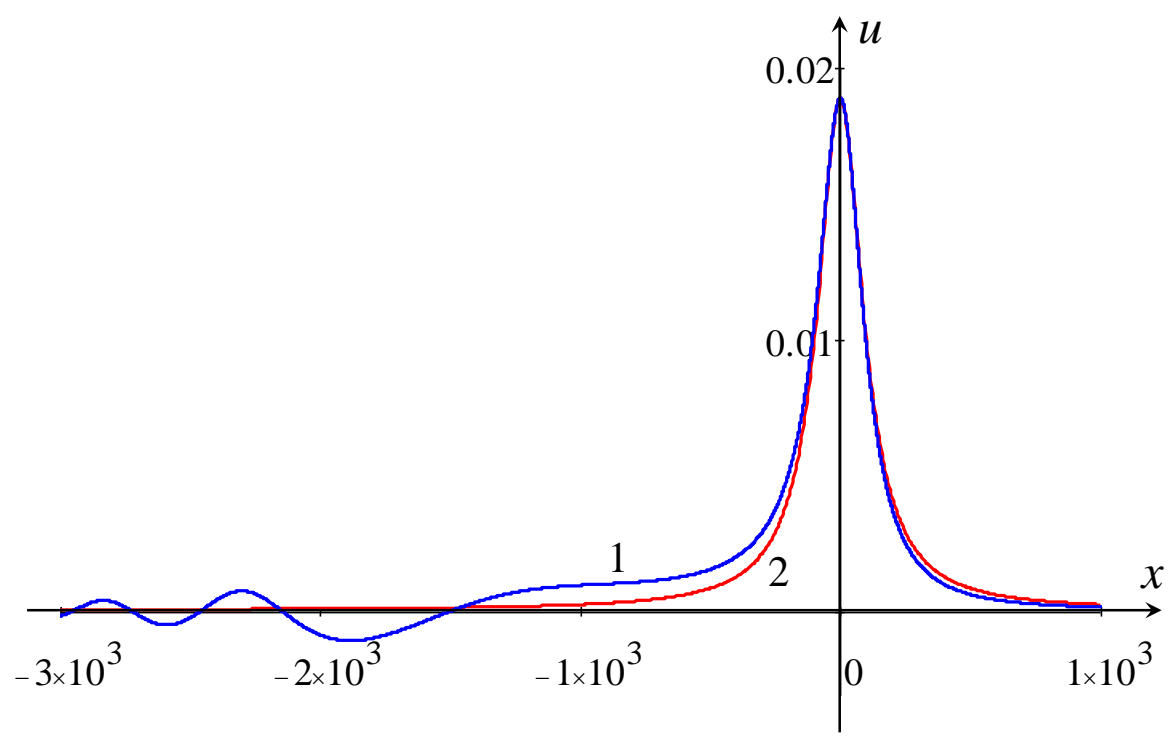

Figure 4: (colour online). Solitary wave profile at $t=60,000(t / \tau=3,000)$ (line 1$)$ for the BO equation (4) with Reynolds dissipation. Line 2 represents a BO soliton of the same amplitude as the leading pulse shown by line 1 . The parameters are $\alpha=2, \beta=1$ and $\delta=0.1$.

as

$$
\frac{\partial u}{\partial t}+\alpha u \frac{\partial u}{\partial x}+\frac{\beta}{\pi} \frac{\partial^{2}}{\partial x^{2}} \wp \int_{-\infty}^{+\infty} \frac{u(\xi, t)}{\xi-x} d \xi-\frac{\delta}{\pi} \frac{\partial}{\partial x} \wp \int_{-\infty}^{+\infty} \frac{u(\xi, t)}{\xi-x} d \xi=0
$$

The energy balance equation (9) determining the evolution of the soliton parameters is then

$$
\frac{d E}{d t}=\frac{\delta}{\pi}\left\langle u(x, t) \frac{\partial}{\partial x} \wp \int_{-\infty}^{+\infty} \frac{u(\xi, t)}{\xi-x} d \xi\right\rangle,
$$

where the angular brackets stand for integration in $x$ over the infinite domain. The expression in the right hand side can be directly evaluated, yielding

$$
\frac{\delta}{\pi}\left\langle u(x, t) \frac{\partial}{\partial x} \wp \int_{-\infty}^{+\infty} \frac{u(\xi, t)}{\xi-x} d \xi\right\rangle=-\frac{\pi}{4} \delta A^{2} .
$$

Then using the expression for the soliton energy $E_{s}(14)$, we obtain

$$
\frac{d A}{d t}=-\frac{\delta \alpha A^{2}}{4 \beta}
$$

whose solution is

$$
A(t)=\frac{A_{0}}{1+t / \tau}, \quad \tau=\frac{4 \beta}{\delta \alpha A_{0}}=\frac{\Delta_{0}}{\delta} .
$$


The asymptotic dependence of the soliton amplitude on the normalised time is shown in Fig. 5 by line 1. A simple estimate of the relative strength of the dissipative term $(\sim \delta A(t) / \Delta(t))$ to the dispersive or nonlinear terms (see Sect. 2) gives $\delta \Delta(t) / \beta \sim \delta(1+t / \tau) / \alpha A_{0}$. This ratio gradually increases with time, so that the adiabatic theory should become less valid as time increases. However, the solitary wave profile exactly coincides with the profile of a $\mathrm{BO}$ soliton of the same amplitude for all times and no shelf behind the leading solitary wave is generated (see, e.g., Fig. 6). Such good agreement between the numerical data and asymptotic theory suggests that a soliton solution of the form

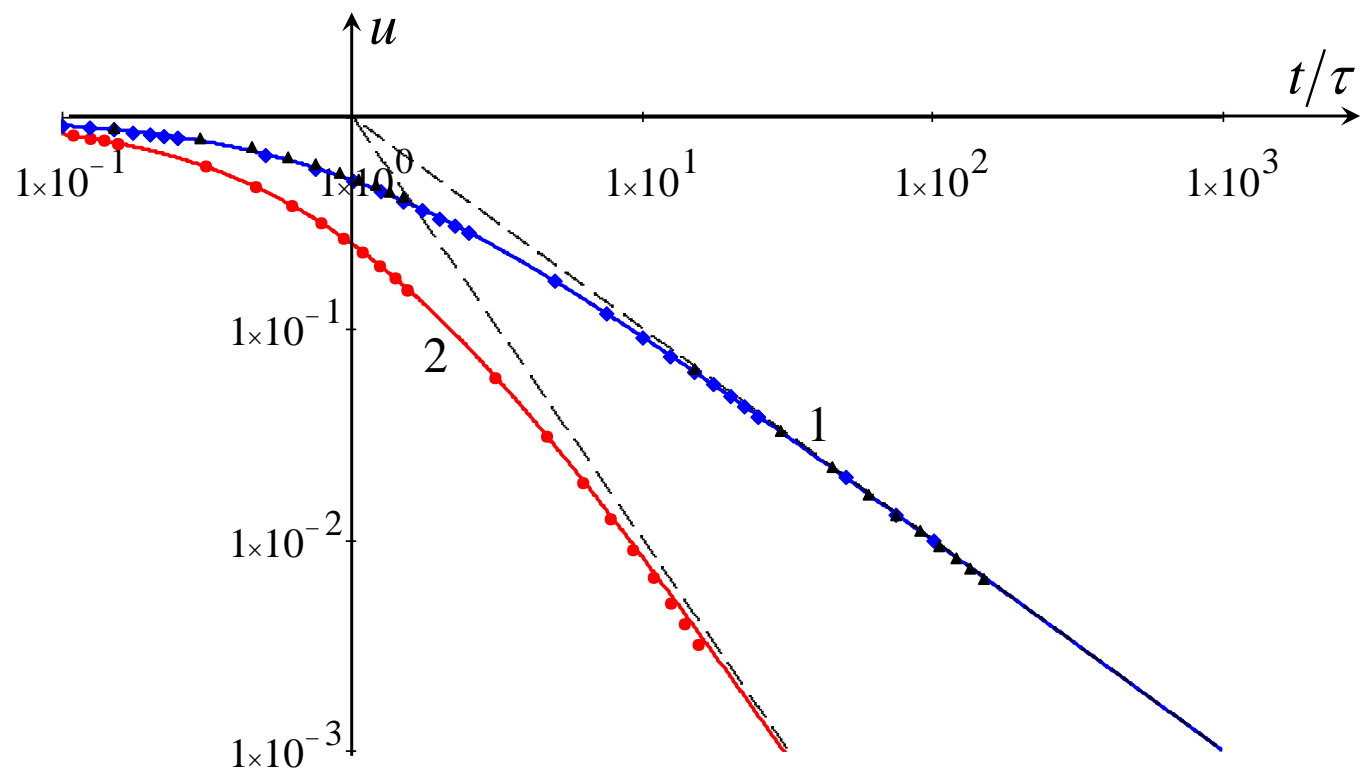

Figure 5: (colour online). Soliton amplitude dependences (log-log scale) on the normalised time $t / \tau$ in the cases of Landau damping $(\delta=0.01)$ and Chezy friction $(\delta=0.01)$ (line 1), and dissipation in a laminar boundary layer $(\delta=0.001)$ (line 2). Dashed lines show the asymptotic dependences at large time $(t \rightarrow \infty): u \sim(t / \tau)^{-1}$ for line 1 and $u \sim(t / \tau)^{-2}$ for line 2, respectively. Symbols show the numerical data; rhombuses for Landau damping and triangles for Chezy friction (not all data is shown to avoid overlapping of symbols). The other parameters are $\alpha=2$ and $\beta=1$.

$$
u(x, t)=\frac{A(t)}{1+\xi^{2} / \Delta^{2}(t)}, \quad \text { where } \quad \xi=x-\int V(t) d t
$$

can be an exact solution of Eq. (26), provided that $A(t)$ is given by Eq. (30), $\Delta=4 \beta / \alpha A(t)$ and $V(t)=\alpha A(t) / 4$. Direct substitution of this solution into Eq. 26) confirms that this is the case. 


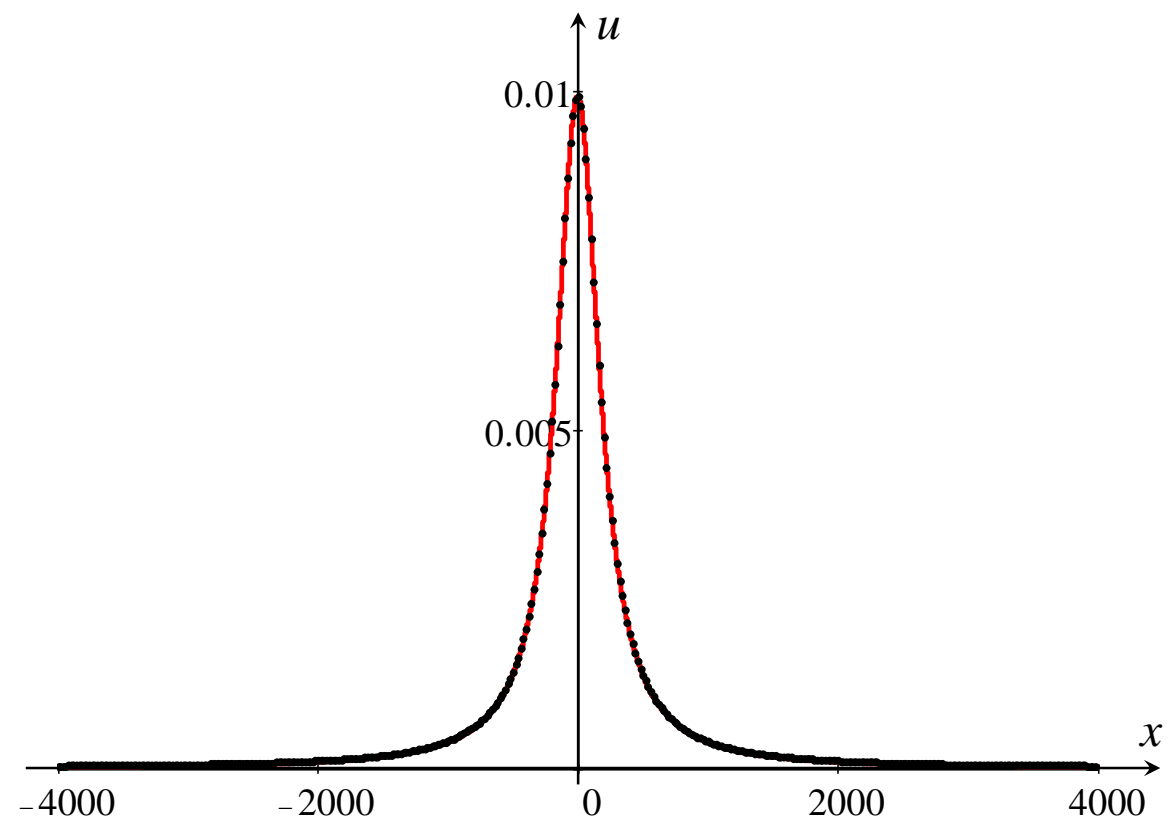

Figure 6: (colour online). Solitary wave profile at $t=20,000(t / \tau=100)$ in the case of Landau damping. Dots show the numerical data for $\alpha=2, \beta=1$ and $\delta=0.01$.

This is a nice example of an exact non-stationary solution in the form of a decaying soliton of a nonlinear equation with dissipation. It is worth recalling that for Landau damping the total wave mass is conserved, based on Eq. (4). As the soliton mass is a constant, then formally no trailing shelf can be formed at leading order. This implies that the adiabatic theory will hold for large time. In fact, it is valid for all time in the case of Landau damping.

A linear wave governed by the damped BO Eq. (4) decays exponentially $u_{\text {lin }} \sim \exp (-\delta k t)$, where $k$ is the wavenumber. For a $\mathrm{KdV}$ soliton, the amplitude decay due to Landau damping is more rapid [26, 27], $A(t)=A_{0}(1+t / \tau)^{-2}$, due to the specific relationship between the soliton width $\Delta$ and amplitude $A$.

\section{Soliton decay due to dissipation in a laminar boundary layer}

In some flows a pycnocline (a sharp density interface in a stratified fluid) may be located near a boundary, so that the near-bottom layer is relatively thin and overlayed by a very deep upper layer. Long (in comparison with the thickness of the lower layer) internal waves propagating on a 
pycnocline are described by the $\mathrm{BO}$ equation. In these situations, wave dissipation can be caused by a laminar boundary layer or wave scattering on random bottom irregularities. In the former case the model equation is the BO equation (4) with the linear operator $\mathcal{D}[u](5)$ with $m=1 / 2$, whereas in the latter case the appropriate model is equation (4) with nonlinear Chezy friction described by the operator $\mathcal{D}[u]=|u| u$. In this section we shall study soliton decay caused by dissipation in a laminar boundary layer, and in the next section decay due to Chezy friction.

The dissipative function $F$ in the case of dissipation in a laminar boundary layer can be calculated with the help of Eq. (12) with $m=1 / 2$, to yield

$$
F=\frac{8 \sqrt{2}}{\pi} \int_{0}^{+\infty} \sqrt{k}\left(\frac{\pi \beta}{\alpha}\right)^{2} e^{-2 k \Delta} d k=\frac{\pi}{4} \sqrt{\frac{\pi \beta}{\alpha}} A^{3 / 2} .
$$

Then, using the energy balance equation (9) and expression (14) for the soliton energy, we can calculate the dependence of the soliton amplitude on time

$$
A(t)=\frac{A_{0}}{(1+t / \tau)^{2}}, \quad \text { where } \quad \tau=\frac{8}{\delta} \sqrt{\frac{\beta}{\pi \alpha A_{0}}}=\frac{4}{\delta} \sqrt{\frac{\Delta_{0}}{\pi}} .
$$

The dependence of the soliton amplitude on the normalised time is shown in Fig. 5 by line 2. The total wave mass is conserved within Eq. (4) with the appropriate decay term. A linear wave in this case decays exponentially, $u_{\text {lin }} \sim \exp (-\delta \sqrt{k} t)$, where $k$ is the wavenumber. A simple estimate of the relative strength of the dissipative term $\left(\sim \delta A(t) / \Delta^{3 / 2}(t)\right)$ in comparison with the nonlinear and dispersive terms (see Sect.22) shows that the ratio of these terms is $\delta / \sqrt{\alpha \beta A(t)}=\delta(1+t / \tau) / \sqrt{\alpha \beta A_{0}}$, which increases with time. This means that the asymptotic theory ceases to be valid when this ratio is unity, i.e., when $t / \tau \approx \sqrt{\alpha \beta A_{0}} / \delta$. For $\delta=10^{-3}, \alpha=2$ and $\beta=A_{0}=1$, this gives $t / \tau \approx \sqrt{2} \times 10^{3}$.

Figure 7 illustrates the deformation of the solitary wave profile in the course of dissipation. In this case mass is conserved too. Therefore, no trailing shelf can be formed at leading order. This again implies that the adiabatic theory will hold for a long time. However, solution (2) with the amplitude decaying as per Eq. (33) is not an exact solution in this case.

For a $\mathrm{KdV}$ soliton the amplitude decay due to dissipation in a laminar boundary layer is more rapid [8], $A(t)=A_{0}(1+t / \tau)^{-4}$, due to the specific relationship between the soliton width $\Delta$ and amplitude $A$. 

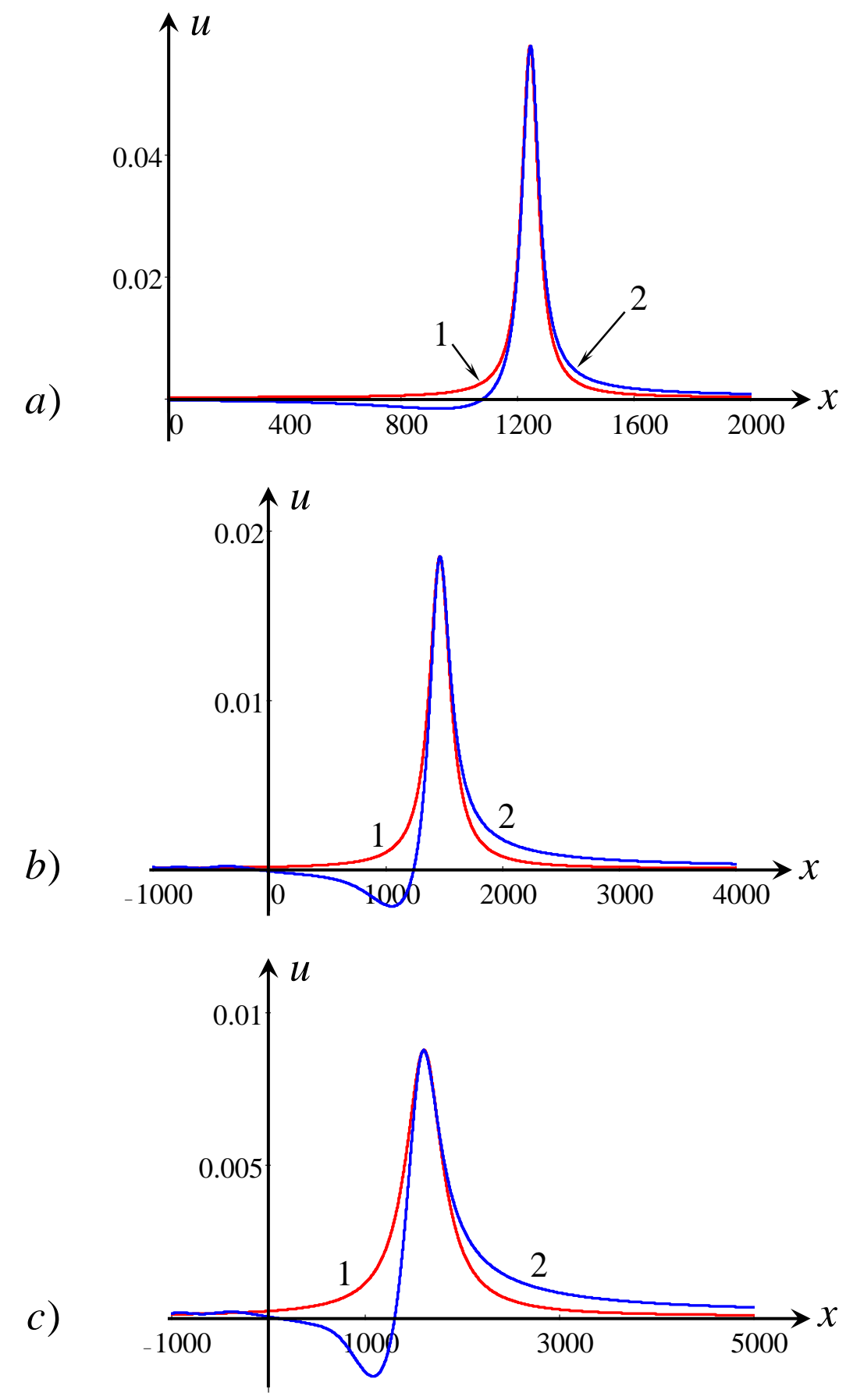

Figure 7: (colour online). Soliton damping due to dissipation in a laminar boundary layer. Solitary wave profiles (lines 2) at different instants of time compared with BO solitons of the same amplitudes (lines 1): a) $t=10,000$ $(t / \tau=3.133)$; b) $t=20,000(t / \tau=6.267)$; c) $t=30,000(t / \tau=9.4)$. Other parameters are $\alpha=2, \beta=1$ and $\delta=0.001$ 


\section{Soliton decay due to Chezy friction}

As was mentioned above, in the case of nonlinear waves propagating on a pycnocline located near a bottom in a deep fluid (for example, in the two layer model of the atmosphere or ocean) Chezy friction can be important. In general, Chezy friction is used as an empirical model to describe wave energy losses due to scattering on random bottom roughness (see, e.g., Ref. [35]). In this case the damped BO Eq. (4) contains the dissipation coefficient $\delta|u|$ which is not a constant, but depends on the modulus of the wave field, and the value of $\delta$ depends on the degree of roughness of the bottom [8]. The energy balance equation is now

$$
\frac{d E}{d t}=-\delta \int_{-\infty}^{+\infty}|u| u^{2} d x=-\frac{3 \pi \delta}{8} A^{3} \Delta=-\frac{3 \pi \beta \delta}{2 \alpha} A^{2} .
$$

Here, we have substituted the expression for $u$ in the form of a BO soliton (4) and used the relationship between its width $\Delta$ and amplitude $A$. From this energy equation we obtain the soliton amplitude evolution

$$
\frac{d A}{d t}=-\frac{3}{2} \delta A^{2} \quad \text { and } \quad A(t)=\frac{A_{0}}{1+t / \tau}
$$

where $\tau=2 /\left(3 \delta A_{0}\right)$. The dependence of the soliton amplitude on the normalised time is the same as in the case of Landau damping (cf. Eq. (30) ) and is shown in Fig. 5 by line 1. In the same figure we present the numerical data (shown by triangles), which is in a good agreement with the theoretical prediction. In Fig. 8 the deformation of soliton profile at different instances of time is shown.

The range of validity of the adiabatic theory can be estimated again either by comparison of the relative strength of the dissipative term $\left(\sim \delta A^{2}(t)\right)$ to the nonlinear and dispersive terms (see Sect. 2), or with the help of the mass balance equation (7). In the former case, if we estimate the ratio of the dissipative to the nonlinear terms, we obtain $t / \tau \approx \alpha^{2} A_{0} / 8 \beta \delta$ (in dimensional variables $t \approx \alpha^{2} / 12 \beta \delta^{2}$ is independent of the initial soliton amplitude). Using the same parameters as above, we obtain $t / \tau \approx 50$. However, surprisingly the numerical data demonstrates very good agreement with the asymptotic theory, even for $t / \tau=300$ (see the triangles in Fig. 5), whereas the solitary wave profile notably differs from the profile of a BO soliton (see Fig. 8). 
a)

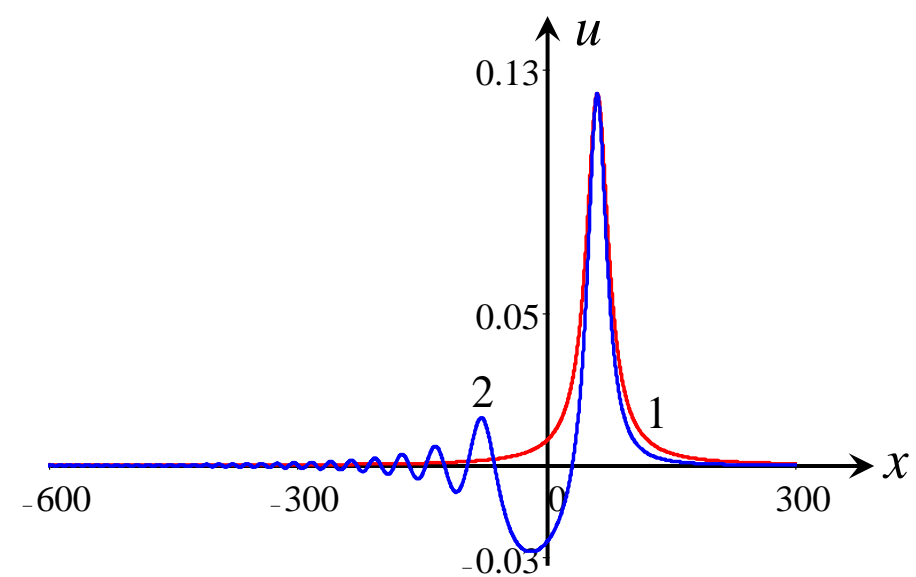

b)

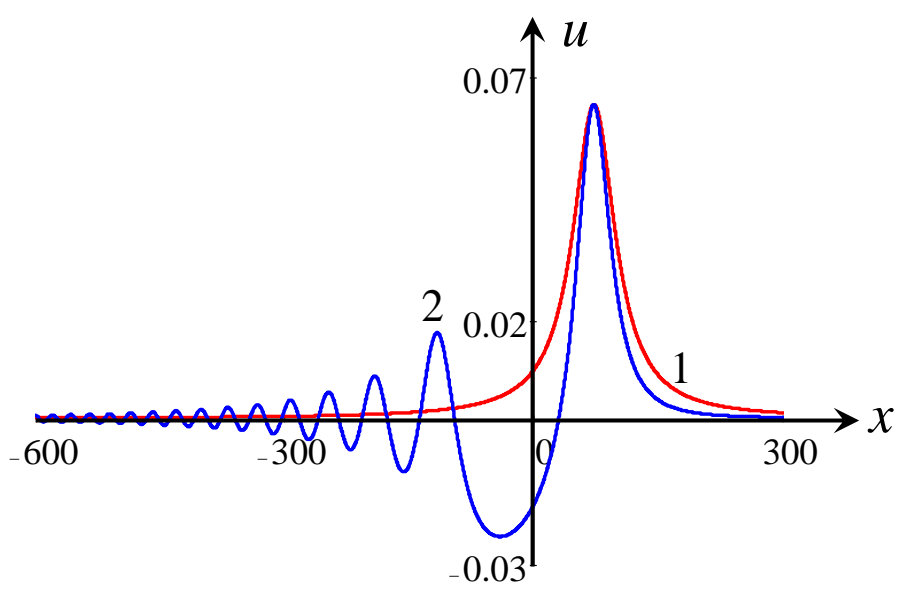

c)

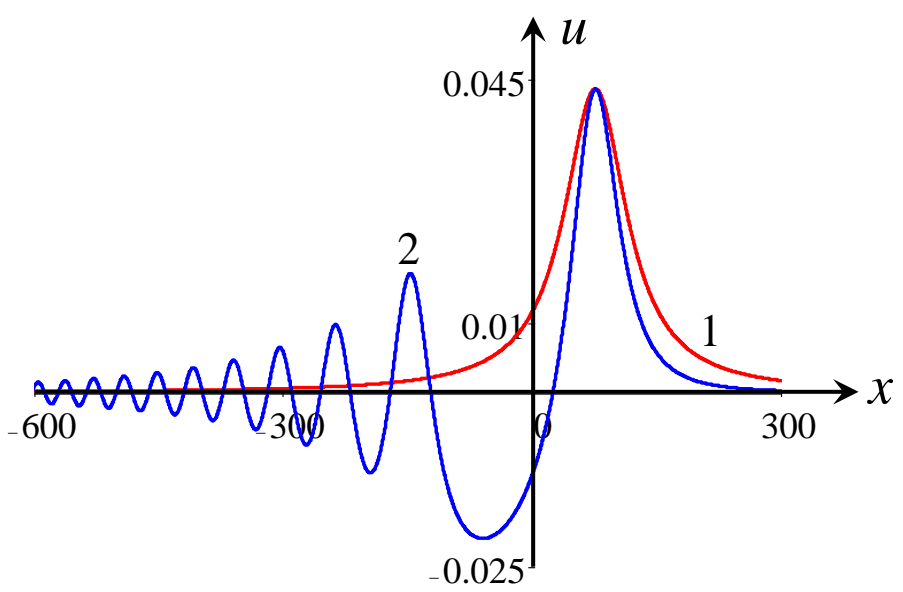

Figure 8: (colour online). Soliton damping due to Chezy friction. Wave profiles (lines 2) at different instants of time compared with BO solitons of the same amplitudes (lines 1): a) $t=500(t / \tau=7.5)$; b) $t=1000(t / \tau=15$; c) $t=1,500(t / \tau=22.5)$. The other parameters are $\alpha=2, \beta=1$ and $\delta=0.01$. 
As mentioned, another estimate of the range of validity of the asymptotic theory follows from the mass balance equation, which can be presented in the form

$$
\frac{d M_{\text {shelf }}}{d t}+\frac{d M_{s}}{d t}=-\delta \int_{-\infty}^{+\infty}|u| u d x=-2 \delta E_{s} .
$$

Bearing in mind again that $d M_{s} / d t=0$ for a BO soliton and $d M_{\text {self }} / d t=u_{\text {shelf }} V(t)$, we obtain (cf. Eq. (19p)

$$
u_{\text {shelf }}=-2 \delta \frac{E_{s}}{V(t)}=-8 \pi \frac{\beta \delta}{\alpha^{2}} .
$$

Remarkably the shelf is independent of the solitary wave amplitude and is a constant. The adiabatic theory breaks down when the soliton amplitude $A(t)$ decreases to this value. Then using Eq. (35), we finally obtain $t / \tau \approx \alpha^{2} A_{0} / 8 \pi \beta \delta$ as an estimate of when the asymptotic theory ceases to be valid. This gives, however, a slightly worse estimate for the limit of validity of the asymptotic theory compared with that obtained above. This is due to the factor $\pi$ in the denominator. In any case, both estimates show that the breakdown of the adiabatic theory is rather slow in the case of Chezy decay.

When the shelf forms it obeys long wave dynamics initially, so that it obeys the approximate equation

$$
\frac{\partial u_{\text {shelf }}}{\partial t}+\delta\left|u_{\text {shelf }}\right| u_{\text {shelf }} \approx 0 \quad \text { and } \quad u_{\text {shelf }} \approx-\frac{1}{t+F(x)},
$$

where $x=P(t)$ defines $t=R(x)$. The arbitrary function $F(x)$ can be evaluated by requiring that $u_{\text {shelf }}(t-R(x))$ is given by Eq. (37) at $t=R(x)$. That is,

$$
\frac{18 \pi \beta \delta}{\alpha^{2}}=\frac{1}{R(x)+F(x)}, \quad u_{\text {shelf }} \approx-\frac{1}{t-R(x)+\alpha^{2} /(8 \pi \beta \delta)} .
$$

Thus, the shelf again exists in $x<P$, but now decays only algebraically behind the solitary wave (cf. Eq. (22)).

Note also that the character of BO soliton decay in this case is similar to that obtained earlier for a $\mathrm{KdV}$ soliton [8]. In both these cases the characteristic time of soliton decay $\tau$ depends only on the initial amplitude and dissipation parameter $\delta$, whereas in some other cases (e.g., in the cases of Reynolds dissipation or Landau damping) it depends also on the nonlinear, $\alpha$, and dispersion, $\beta$, coefficients. 


\section{Influence of large-scale dispersion on soliton decay}

In this section we consider the effect of large scale dispersion on the dynamics of $\mathrm{BO}$ solitons. The BO equation augmented by large scale dispersion was derived by Grimshaw [7] to describe internal waves in a rotating fluid. It can be presented in a form similar to the Ostrovsky equation [10, 23] derived for shallow water waves

$$
\frac{\partial u}{\partial t}+\alpha u \frac{\partial u}{\partial x}+\frac{\beta}{\pi} \frac{\partial^{2}}{\partial x^{2}} \wp \int_{-\infty}^{+\infty} \frac{u(\xi, t)}{\xi-x} d \xi=\gamma v, \quad u=\frac{\partial v}{\partial x}, \quad v=-\int_{x}^{\infty} u(x, t) d x
$$

Here, $\gamma$ is a parameter characterising the large scale dispersion. In the context of internal waves in a rotating ocean $\gamma=f^{2} / 2 c$, where $f$ is the Coriolis parameter (see [2, 7]).

For the derivation of the energy balance equation we multiply the perturbed BO Eq. (40) by $u$ and integrate over $x$, assuming that ahead of the soliton there is no wave perturbation, i.e. $u(x, t) \rightarrow$ 0 as $x \rightarrow \infty$. We then obtain

$$
\frac{d E}{d t}=-\gamma \int_{-\infty}^{+\infty} u(x, t)\left(\int_{-\infty}^{x} u(\xi, t) d \xi+C\right) d x
$$

where $C$ is chosen such that the aforementioned condition at $x=\infty$ is satisfied. Substituting the soliton solution (2) and choosing $C=-\pi / 2$, after simple manipulations we obtain

$$
\frac{d A}{d t}=-\frac{8 \pi \gamma \beta}{\alpha}
$$

which gives

$$
A(t)=A_{0}\left(1-\frac{t}{\tau}\right), \quad \tau=\frac{\alpha A_{0}}{8 \pi \beta \gamma}=\frac{1}{2 \pi \gamma \Delta_{0}},
$$

where $\Delta_{0}$ is the initial soliton width.

The dependence of the amplitude $A(t)$ on time is depicted in Fig. 9 in which the asymptotic result (43) is compared with numerical solutions for various values of the parameter $\gamma$. This figure demonstrates that the applicability of the adiabatic theory is very sensitive to the value of this parameter, with small values being required for good agreement with the asymptotic result. As $\gamma$ decreases, the time required for significant decay greatly increases as the half-life of the soliton increases. Indeed, for $\gamma=10^{-6}$, the numerical results shown in Figure 9 are up to $t=50,000$. 


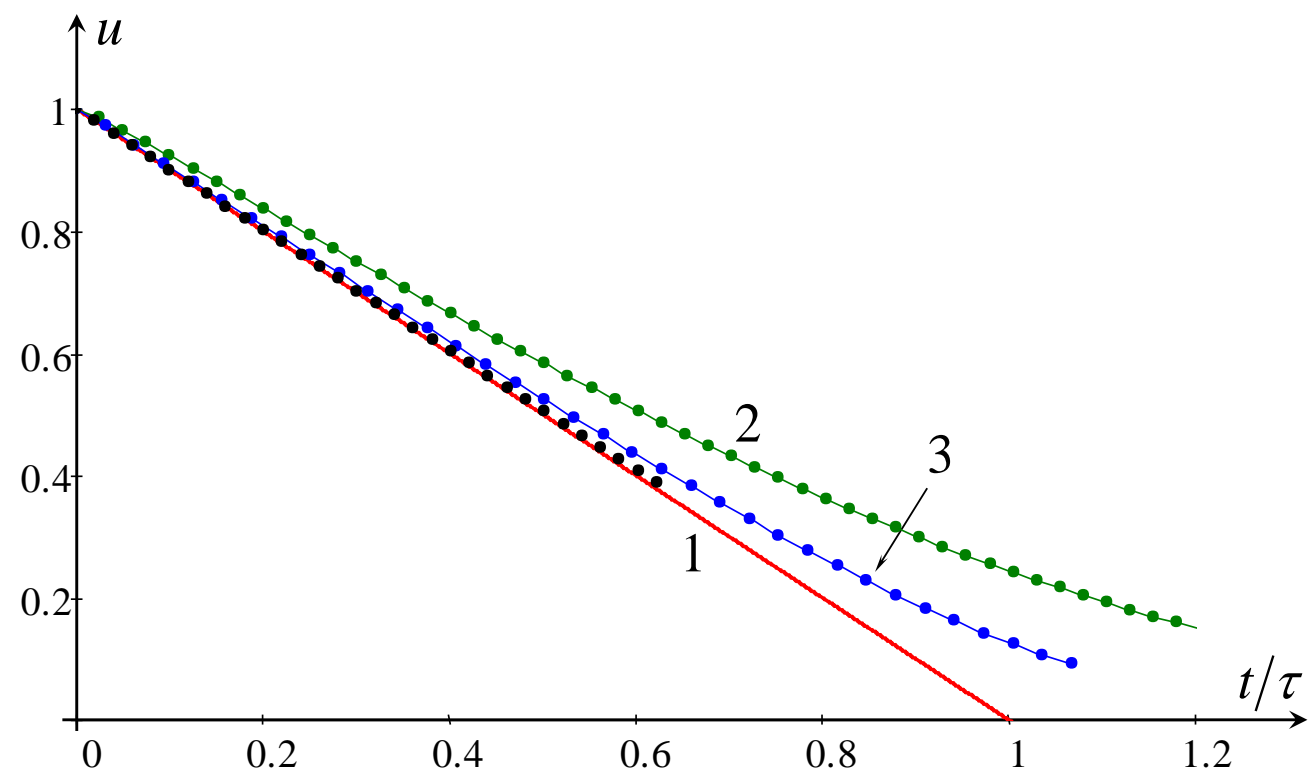

Figure 9: (colour online). Soliton amplitude dependence on the normalised time $t / \tau$ for the radiative decay caused by large-scale dispersion (line 1). Symbols represent numerical data obtained with $\alpha=2, \beta=1$, and $\gamma=10^{-4}$ (line 2), $\gamma=10^{-5}$ (line 3$)$, and $\gamma=10^{-6}($ dots on line 1$)$.

A simple estimate of the relative strength of the large scale dispersive term $(\sim \gamma v \sim \gamma A(t) \Delta(t))$ in comparison with the small scale dispersive term $\left(\sim \beta A(t) / \Delta^{2}(t)\right)$ shows that their ratio is proportional to

$$
\frac{\gamma}{\beta}\left(\frac{4 \beta}{\alpha A(t)}\right)^{3}=\frac{\gamma}{\beta}\left(\frac{4 \beta}{\alpha A_{0}}\right)^{3} \frac{1}{(1-t / \tau)^{3}} .
$$

This ratio increases with time and leads to the breakdown of the asymptotic theory. Formally this happens when

$$
\frac{t}{\tau} \approx 1-\frac{4 \beta}{\alpha A_{0}}\left(\frac{\gamma}{\beta}\right)^{1 / 3} .
$$

For $\gamma=10^{-4}, t / \tau \approx 0.91$, whereas for $\gamma=10^{-5}, t / \tau \approx 0.96$ (the other parameters are as in Fig. 9).

Note that in the case of the $\mathrm{KdV}$ equation the soliton amplitude also decays to zero in a finite time, but in accordance with the quadratic law $A(t)=A_{0}(1-t / \tau)^{2}$ [9, [10]. As can be seen from Fig. 9, the numerical solutions deviate from the theoretical prediction and the extent of the difference depends on the parameter $\gamma$. Even for the extremely small value of $\gamma=10^{-5}$ the deviation is notable and gradually increases with time (see line 3). Figure 10 illustrates a soliton profile in the 
process of adiabatic decay. The soliton is accompanied by the radiation of a large scale trailing wave. A similar structure has been found for a KdV soliton [9], which is expected.

a)

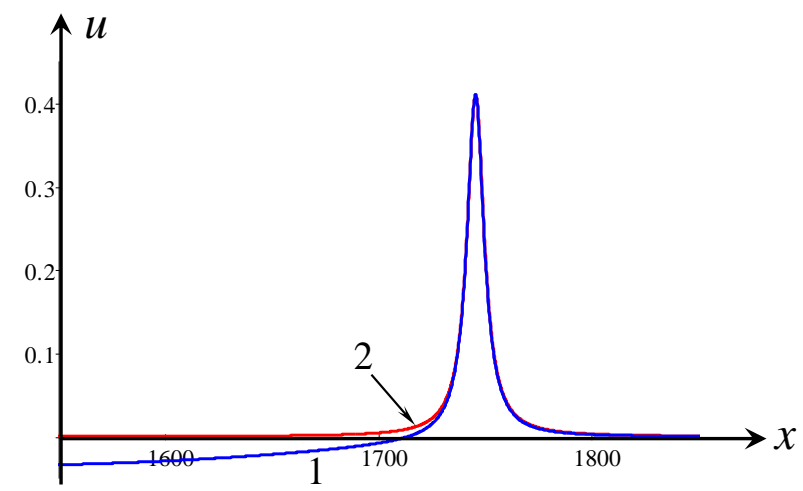

b)

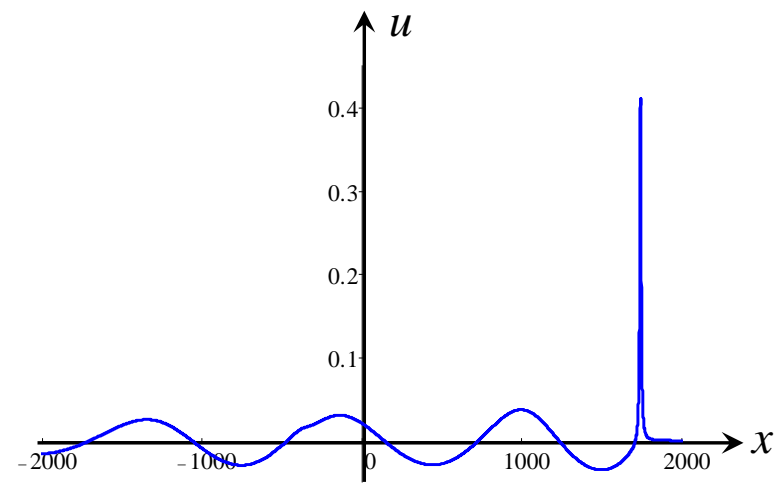

Figure 10: (colour online). Soliton profile at $t=5,000(t / \tau=0.63)$ for adiabatic decay under the influence of radiative losses within the framework of Eq. 40 with $\gamma=10^{-5}, \alpha=2$ and $\beta=1$. (a) leading solitary wave (line 1) compared with a BO soliton of the same amplitude (line 2); (b) leading solitary wave shown together with the generated periodic trailing wave.

The structure of the trailing wave seen in Fig. 10(b) can be calculated with the help of asymptotic theory [9]. To this end, let us present a solution of Eq. 40] as an asymptotic expansion, assuming that $\gamma$ is a small parameter

$$
u=u_{0}+u_{1}+\ldots,
$$

where $u_{0}$ is a BO soliton (2) travelling at speed $V$ in the $x$ direction and $u_{1}$ is of order of $\gamma$. Substituting this expansion into the damped BO Eq. (40), we obtain at leading order in the small 
parameter $\gamma$

$$
-V \frac{\partial u_{1}}{\partial X}+\alpha \frac{\partial\left(u_{0} u_{1}\right)}{\partial X}+\frac{\beta}{\pi} \frac{\partial^{2}}{\partial X^{2}} \wp \int_{-\infty}^{\infty} \frac{u_{1}(\xi, t)}{\xi-x} d \xi=\gamma v_{0}-\frac{\partial u_{0}}{\partial T}, \quad v_{0}=-\int_{X}^{\infty} u_{0} d x
$$

Here $\partial u_{0} / \partial T$ is the slow time evolution of the soliton

$$
u_{0}(X, T)=\frac{A(T)}{1+X^{2} / \Delta^{2}(T)}, \quad X=x-P(t), \quad \frac{\partial P}{\partial T}=V(T)=\frac{\alpha A(T)}{4}=\beta \Delta(T) .
$$

The homogeneous equation for Eq. (47) has a solution $u_{1}=\partial u_{0} / \partial X$. The necessary compatibility condition to remove the secular growing term at this order is then

$$
\int_{-\infty}^{\infty}\left(\gamma v_{0}-\frac{\partial u_{0}}{\partial T}\right) u_{0} d X=0
$$

This yields

$$
\frac{d E_{s}}{d T}=-\gamma\left[v_{0}^{2}\right]_{-\infty}
$$

where $E_{s}$ is the soliton energy. Of course, this equation is the same as Eq. (41). Therefore, at leading order in the parameter $\gamma$ we obtain Eq. (43) for the soliton amplitude.

Then, bearing in mind that a soliton vanishes at infinity, we obtain from Eq. 47) that as $X \rightarrow$ $-\infty$,

$$
-V \frac{\partial u_{1}}{\partial X} \approx \gamma v_{0}, \quad v_{0} \approx-\pi A \Delta=-\frac{4 \pi \beta}{\alpha}
$$

in the reference frame moving with the soliton velocity $V$. It then follows from Eq. (51) that the solution for $u_{1}$ grows secularly as $x \rightarrow-\infty$

$$
u_{1} \sim \frac{16 \pi \beta \gamma}{\alpha^{2} A} X
$$

This shelf term can then be matched at $X<0$ to a trailing sinusoidal wave of wavenumber $\kappa$,

$$
u_{\text {shelf }}=A_{\text {shelf }} \sin (\kappa X) \text {, }
$$

where $\kappa^{2}=\gamma / V$ and $A_{\text {shelf }} \kappa=16 \pi \beta \gamma / \alpha^{2} A$. Therefore $A_{\text {shelf }}=\left(8 \pi \beta / \alpha^{2}\right) \sqrt{\alpha \gamma / A}$.

Note that as the main wave decays, this shelf grows, eventually invalidating the asymptotic expansion 46 when $\alpha A \sim \sqrt[3]{(8 \pi \beta)^{2} \gamma}$. Thus the adiabatic theory breaks down quite quickly. 
The approximate shelf solution $(53)$ is valid in the intermediate stage of soliton decay when the radiation field is already well formed after a transient period, but the solitary wave amplitude is still much greater than the amplitude of the trailing wave, $\alpha A \gg 4 \sqrt[3]{\pi^{2} \beta^{2} \gamma}$. For the parameters used in Fig. 9, this means that $A \gg 0.09$, which is in agreement with the comparisons shown. Figure 11 illustrates comparisons of the wave field behind the leading solitary wave as obtained in numerical calculations (line 1) and that from Eq. (53) (line 2). The adiabatic theory gives that the solitary wave completely vanishes in a finite time $\tau$, see (43). However, the long term behaviour is that the leading wave transforms into a quasi-sinusoidal wavetrain which can be described by the generalised nonlinear Schrödinger (NLS) equation [12, 13]. We shall derive this equation below and give its stationary solution in the form of an envelope soliton.

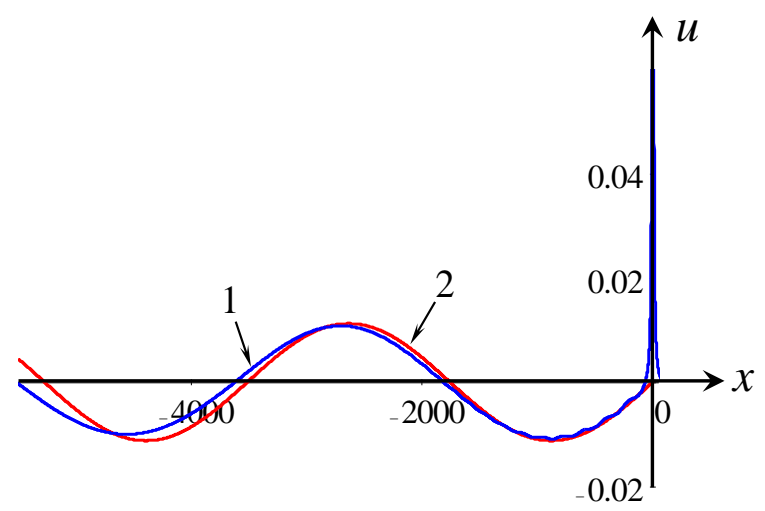

Figure 11: (colour online) Trailing wave behind a solitary wave for large scale dispersion as obtained from numerical calculations (line 1) and theoretically predicted by Eq. (53) (line 2) at $t=30,000(t / \tau=0.377)$. The parameters are $\alpha=2, \beta=1$ and $\gamma=10^{-6}$.

\subsection{Extended nonlinear Schrödinger equation}

The nonlinear Schrödinger equation is a weakly nonlinear equation describing the interaction of a wave packet grouped around a central wavenumber. As such, it arises in a large number of physical applications, including fluid mechanics and nonlinear optics [1, 15, 32]. This equation is

$$
i\left(\frac{\partial A}{\partial t}+c_{g} \frac{\partial A}{\partial x}\right)+\mu \frac{\partial^{2} A}{\partial x^{2}}+v|A|^{2} A=0 .
$$

This equation is for the complex envelope $A(x, t)$ of the weakly nonlinear solution

$$
u=A(x, t) \exp (i k-i \omega t)+\text { c.c. }+\cdots,
$$


where c.c. stands for the complex conjugate. Here $\omega(k)=-\beta k^{2}+\gamma / k$ is the linear dispersion relation. At leading order the envelope $A$ moves with the linear group velocity $c_{g}=d \omega / d k=$ $-2 \beta k-\gamma / k^{2}$. The dispersive term in Eq. 54 generically has the coefficient $\mu=(1 / 2)\left(d c_{g} / d k\right)$, but the coefficient $v$ of the cubic nonlinear term is system dependent.

Our concern here is with the situation when $\mu=0$, selecting a wavenumber $k=k_{m}$ for which the group velocity has a local extremum. In this case we must replace the standard NLS equation (54) with the generalised one including the next higher order (cubic) dispersion term

$$
i\left(A_{t}+c_{g} A_{x}\right)+\mu A_{x x}+i \mu_{1} A_{x x x}+v|A|^{2} A=0,
$$

where $\mu_{1}=-(1 / 6)\left(d^{2} c_{g} / d k^{2}\right) \neq 0$ is the coefficient of this third order linear dispersive term. Note that we retain $\mu$, even although it may be zero, in order to broaden the parameter space. Equations of this type arise in nonlinear optics [3, 17, 19] where it has been found advantageous to include in addition the next order nonlinear terms. Thus, we extend Eq. (56) (details are given in the Appendix) to

$$
i\left(A_{t}+c_{g} A_{x}\right)+\mu A_{x x}+i \mu_{1} A_{x x x}+v|A|^{2} A+i\left(v_{1}|A|^{2} A_{x}+v_{2} A^{2} A_{x}^{*}\right)=0
$$

Here the ${ }^{*}$ superscript denotes the complex conjugate. Technically the terms with coefficients $v_{1}$ and $v_{2}$ are higher order, but nevertheless they are needed, as will be shown below.

We seek a solitary wave solution of Eq. (57) in the form

$$
A=F(X-V t) \exp (i \kappa X-i \sigma t), \quad X=x-c_{g} t
$$

where we choose the gauge $\kappa$ and the chirp $\sigma$ so that

$$
v+2 \kappa v_{2}=\frac{\left(v_{1}+v_{2}\right) \mu}{3 \mu_{1}}, \quad \sigma=3 \kappa V+8 \mu_{1} \kappa^{3}+\frac{\mu}{\mu_{1}}\left(4 \mu_{1} \kappa^{2}-V\right)-\frac{2 \kappa \mu^{2}}{\mu_{1}} .
$$

Note that when $\mu=0, \kappa=-v / 2 v_{2}$. Then $F(X)$ is real valued and satisfies the equation

$$
\mu_{1} F_{X X}-\tilde{V} F+\frac{v_{1}+v_{2}}{3} F^{3}=0, \quad \text { where } \quad \tilde{V}=V+3 \mu_{1} \kappa^{2}-\mu \kappa .
$$

This equation has a sech solitary wave solution, provided that $\tilde{V}>0$ (a suitable choice of $V$ can always achieve this condition) and $\mu_{1}\left(v_{1}+v_{2}\right)>0$. Note that the higher-order nonlinear terms are 
needed to obtain this solution. Explicitly

$$
F=a \operatorname{sech} \frac{X}{\Delta}, \quad \text { where } \quad \tilde{V}=\frac{\mu_{1}}{\Delta^{2}}=\frac{\left(v_{1}+v_{2}\right) a^{2}}{6} .
$$

The development of these envelope solitons from the initial BO soliton can be studied numerically through very long term calculation. However, it is out of the scope of this paper.

To conclude this Section we also consider, for the sake of completeness, the case of negative $\gamma$, which can be realised in physical systems with a positive small scale dispersion. In this case the initial BO soliton does not decay, but gradually evolves into another soliton of a higher amplitude and zero mean value. A similar situation occurs for $\mathrm{KdV}$ solitons which gradually transform into Ostrovsky solitons under the influence of large scale dispersion [14]. In Fig. 12 we present a stationary soliton obtained numerically using the Petviashvili method [28, 29]. Under the influence of large scale dispersion the soliton becomes taller and narrower in comparison with the BO soliton.

Thus, within the framework of the asymptotic theory a BO soliton experiences a terminal decay in accordance with Eq. (43) and completely vanishes in the extinction time $\tau$. However, in reality it does not completely disappear, but transforms into an envelope soliton (61) after a very long time. This process is well known for a KdV soliton within the framework of the Ostrovsky equation

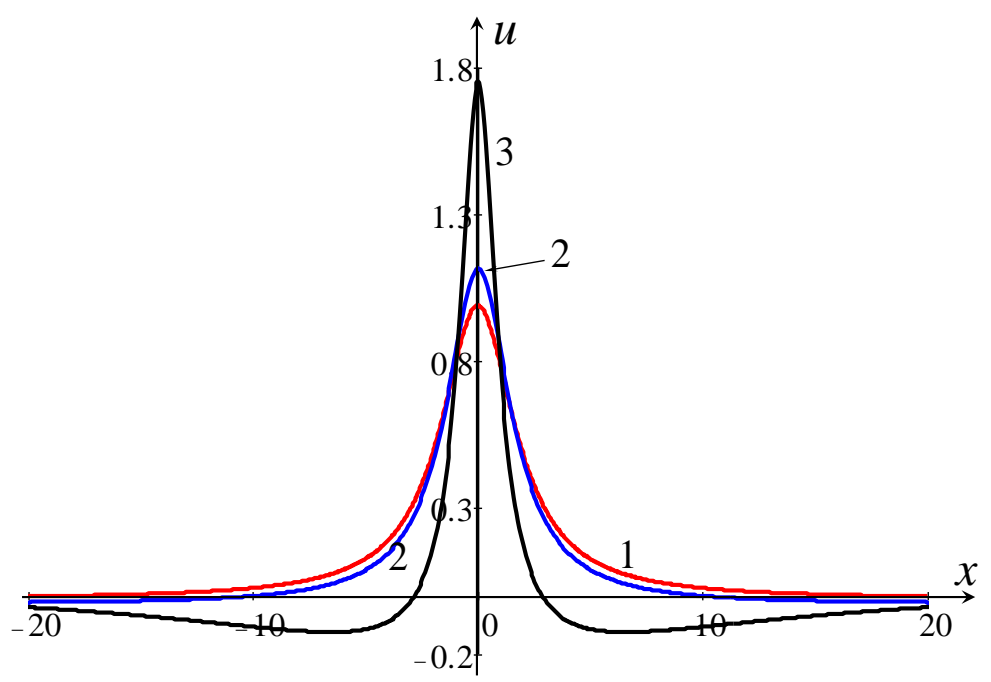

Figure 12: (colour online) BO soliton (line 1) compared with numerical solutions of Eq. 40. for $\gamma=-0.0001$ (line 2) and $\gamma=-0.01$ (line 3). The values of the other parameters are $\alpha=2, \beta=1$ and $V=0.5$. 
$[12,13]$. We do not consider here the long term evolution of a BO soliton into an envelope soliton. This interesting issue will be a matter for a separate study.

\section{Discussion and conclusion}

In this paper we have presented a detailed analysis of the adiabatic decay of a BO soliton under the action of various dissipation mechanisms. Theoretical solutions derived by means of asymptotic theory, which presume the smallness of the dissipation, were compared against numerical solutions. Good agreement between the theory and numerical solutions was found when the dissipative coefficients were sufficiently small. The size of the dissipation coefficients needed for good agreement depended greatly on the exact dissipation mechanism. Estimates of the time scale for the validity of the asymptotic theory for each type of dissipation were found. These were found to be in agreement with numerical results. The results of this study are summarised in Table.

Table. Summary of cases considered and corresponding decay laws for a BO soliton

\begin{tabular}{|l|l|l|l|}
\hline Type of dissipation & Decay character & Characteristic decay time & Equation \\
\hline Rayleigh dissipation & $A(t)=A_{0} e^{-t / \tau}$ & $\tau=\frac{1}{2 \delta}$ & Eq. \\
\hline Reynolds dissipation & $A(t)=\frac{A_{0}}{\sqrt{1+t / \tau}}$ & $\tau=\frac{8 \beta^{2}}{\delta \alpha^{2} A_{0}^{2}}=\frac{\Delta_{0}^{2}}{2 \delta}$ & Eq. \\
\hline Landau damping & $A(t)=\frac{A_{0}}{1+t / \tau}$ & $\tau=\frac{4 \beta}{\delta \alpha A_{0}}=\frac{\Delta_{0}}{\delta}$ & Eq. \\
\hline Decay in a laminar boundary layer & $A(t)=\frac{A_{0}}{(1+t / \tau)^{2}}$ & $\tau=\frac{8}{\delta} \sqrt{\frac{\beta}{\pi \alpha A_{0}}}=\frac{4}{\delta} \sqrt{\frac{\Delta_{0}}{\pi}}$ & Eq. \\
\hline Chezy friction & $A(t)=\frac{A_{0}}{1+t / \tau}$ & $\tau=\frac{2}{3 \delta A_{0}}=\frac{\alpha \Delta_{0}}{6 \beta \delta}$ & Eq. $(35)$ \\
\hline Radiative decay & $A(t)=A_{0}\left(1-\frac{t}{\tau}\right)$ & $\tau=\frac{\alpha A_{0}}{8 \pi \beta \gamma}=\frac{1}{2 \pi \gamma \Delta_{0}}$ & Eq. .43$)$ \\
\hline
\end{tabular}

In particular, it has been found that the solution (37) describing soliton decay due to the Landau damping within the adiabatic theory is actually the exact solution of the $\mathrm{BO}$ equation with the Landau damping (26). 
In the case of the radiative decay of a BO soliton under the influence of large scale dispersion with a positive loss coefficient $\gamma$ the theory predicts the terminal decay of the soliton in finite time. This prediction is in broad agreement with the numerical data, except for times very close to the extinction time $\tau$. Numerical results show that the BO soliton does not completely vanish, but transforms into an envelope soliton which can be described by an extended NLS equation. Such an NLS equation was derived and its soliton solution was obtained in analytic form.

Acknowledgements. The research of R.G. was supported by the Leverhulme Trust, grant No: EM-2015-037, through the award of a Leverhulme Emeritus Fellowship. Y.S. acknowledges the funding of this study from the State task program in the sphere of scientific activity of the Ministry of Education and Science of the Russian Federation (Project No. 5.1246.2017/4.6) and grant of the President of the Russian Federation for state support of leading scientific schools of the Russian Federation (NSH-2685.2018.5).

\section{Appendix. Derivation of extended nonlinear Schrödinger equation}

Here we derive the extended NLS equation (57) from the perturbed BO equation (40) with large scale dispersive loss. The envelope solitary wave solution (61) in terms of the coefficients of equation 40 will then be found. To this end we seek a solution as the asymptotic expansion

$$
u=A e^{i \theta}+\text { c.c. }+A_{2} e^{2 i \theta}+\text { c.c. }+A_{0}+\ldots,
$$

where the phase is $\theta=k x-\omega t$. Here, it is understood that $A(x, t)$ is a slowly varying function of $x$ and $t$. We expect that the second harmonic $A_{2}$ and the zero harmonic (the mean flow term) $A_{0}$ are of $O\left(|A|^{2}\right)$, where $|A| \ll 1$, and are likewise slowly varying.

Substituting the expansion (62) into the perturbed BO equation (40), we find the dispersion relation

$$
D(\omega, k) \equiv \omega k+\beta k^{2}|k|-\gamma=0
$$

at leading order. Then the leading order terms in the coefficient of the first harmonic yields

$$
D\left(\omega+i \frac{\partial}{\partial t}, k-i \frac{\partial}{\partial x}\right) A+\left(k-i \frac{\partial}{\partial x}\right) \mathrm{NL}=0
$$


where the nonlinear term NL is given by

$$
\mathrm{NL}=-\alpha\left(k-i \frac{\partial}{\partial x}\right)\left(A_{2} A^{*}+A_{0} A+\ldots\right) .
$$

Expanding and noting that $D_{\omega}=k$ gives

$$
i\left(A_{t}+c_{g} A_{x}\right)+\mu A_{x x}+i \mu_{1} A_{x x x}+\mathrm{NL}+\ldots=0
$$

where $\mu=(1 / 2)\left(d c_{g} / d k\right)$ and $\mu_{1}=-(1 / 6)\left(d^{2} c_{g} / d k^{2}\right)$, that is

$$
\mu=\operatorname{sign}(k)\left(-\beta+\frac{\gamma}{|k|^{3}}\right)(=0), \quad \mu_{1}=\frac{\gamma}{k^{4}}\left(=\left(\frac{\beta^{4}}{\gamma}\right)^{1 / 3}\right) .
$$

The values in the brackets are those for $|k|=k_{m} \equiv \sqrt[3]{\beta / \gamma}$.

It now only remains to find the nonlinear term NL. First, we note that to leading order

$$
\gamma A_{0}-\frac{\partial^{2} A_{0}}{\partial x \partial t}+\cdots=\alpha \frac{\partial^{2}|A|^{2}}{\partial x^{2}}+\cdots
$$

Hence, $A_{0}$ is two orders of magnitude smaller than $|A|^{2}$, provided that $\gamma \neq 0$, so that it can be neglected unless $\gamma \neq 0$. We note that if $\gamma=0$, then $A_{0}=\alpha|A|^{2} / c_{g}$ and it is of the same order as $|A|^{2}$. Also, if $\gamma \ll 1$, the solution for $A_{0}$ is again of the same order as $|A|^{2}$, but is nonlocal. Henceforth we assume that $\gamma \neq 0$ and of $O(1)$, since then $k_{m}$ is also $O(1)$. Next

$$
D\left(2 \omega+i \frac{\partial}{\partial t}, 2 k-i \frac{\partial}{\partial x}\right) A_{2}=\frac{\alpha}{2}\left(2 k-i \frac{\partial}{\partial x}\right)^{2} A^{2} .
$$

At leading order

$$
D_{2} A_{2}=2 \alpha k^{2} A^{2}, \quad \text { where } \quad D_{2}=D(2 \omega, 2 k)=4 \beta k^{2}|k|+3 \gamma(=7 \gamma) \text {. }
$$

As in (67), the term in brackets is the value at criticality. As we need the next order terms as well, we expand to next order to yield

$$
D_{2} A_{2}=2 \alpha k^{2} A^{2}-2 i \alpha k \frac{\partial A^{2}}{\partial x}-i D_{\omega}(2 \omega, 2 k) \frac{\partial A_{2}}{\partial t}+i D_{k}(2 \omega, 2 k) \frac{\partial A_{2}}{\partial x}+\ldots
$$

In the last two terms on the right hand side we may now substitute the leading order expression for $A_{2}$, resulting in

$$
D_{2} A_{2}=2 \alpha k^{2} A^{2}+i C_{2} \frac{\partial A^{2}}{\partial x}+\ldots,
$$


where

$$
C_{2}=-2 \alpha k+4 \alpha k^{3} \frac{c_{g}(k)-c_{g}(2 k)}{D_{2}}=-\frac{\alpha k\left(2 \beta k^{2}|k|+9 \gamma\right)}{4 \beta k^{2}|k|+3 \gamma}\left(=-\frac{11}{7} \alpha k_{m}\right) .
$$

Hence, on substituting (72) for $A_{2}$ into Eq. (65) for the nonlinear term we find that

$$
\mathrm{NL}=-\alpha k A_{2} A^{*}+i \alpha \frac{\partial\left(A_{2} A^{*}\right)}{\partial x}+\ldots=v|A|^{2} A+i v_{1}|A|^{2} \frac{\partial A}{\partial x}+i v_{2} A^{2} \frac{\partial A^{*}}{\partial x}
$$

with $v=-2 \alpha^{2} k^{3} / D_{2}$.

Note that $\operatorname{sign}(k) v<0$ for all $k$ and so the NLS equation 66 is focussing for $|k|>k_{m}$ and defocussing for $|k|<k_{m}$, where $k_{m}$ was defined at the extremum for which $\mu=0$. This is in sharp contrast to the reduction of the KdV equation to the NLS equation for which the NLS equation is always defocussing. The reason is twofold. First, the presence of rotation has suppressed the mean flow, and hence changed the sign of $v$ from the KdV case. Secondly, it has introduced the critical turning point $|k|=k_{m}$. The higher order coefficients are given by

$$
\begin{gathered}
v_{1}=-\frac{2 \alpha k C_{2}}{D_{2}}+\frac{4 \alpha^{2} k^{2}}{D_{2}}=\frac{2 \alpha^{2} k^{2}\left(10 \beta k^{2}|k|+15 \gamma\right)}{\left(4 \beta k^{2}|k|+3 \gamma\right)^{2}}\left(=\frac{25 \alpha^{2} k_{m}^{2}}{49 \gamma}\right), \\
v_{2}=\frac{2 \alpha^{2} k^{2}}{D_{2}}\left(=\frac{2 \alpha^{2} k_{c}^{2}}{7 \gamma}\right) .
\end{gathered}
$$

Thus, both $v_{1,2}>0$ for all $k$, confirming that an envelope solitary wave given by Eq. (61) exists for $k=k_{m}$.

\section{References}

[1] M. J. Ablowitz \& H. Segur, Solitons and the Inverse Scattering Transform, (SIAM, Philadelphia, 1981).

[2] J. Apel, L. A. Ostrovsky, Y. A. Stepanyants, \& J. F. Lynch, Internal solitons in the ocean and their effect on underwater sound, J. Acoust. Soc. Am., 121, no. 2, 695-722 (2007).

[3] N. Akhmediev \& A. Ankiewicz, Solitons: Non-Linear Pulses and Beams, (Springer US, 1997).

[4] G.B. Arfken \& H.J. Weber, Mathematical Methods for Physicists, (Harcourt, San Diego, 2001).

[5] K. A. Gorshkov \& L. A. Ostrovsky, Interaction of solitons in nonintegrable systems: direct perturbation method and applications, Physica D, 3, 428-438 (1981).

[6] K. A. Gorshkov \& V. V. Papko, Nonadiabatic stage of damping of solitons and the intermediate asymptotics, Radiophys. and Quantum Electronics, 20, 245-248 (1977). 
[7] R. Grimshaw, Evolution equations for weakly nonlinear long internal waves in a rotating fluid, Stud. Appl. Math., 73, 1-33 (1985).

[8] R. Grimshaw, Internal solitary waves, In Environmental Stratified Flows, ed. by R. Grimshaw, (Kluwer, Boston, Chapter 1, 1-27, 2001).

[9] R. Grimshaw, J.-M. He, \& L. A. Ostrovsky, Terminal damping of a solitary wave due to radiation in rotational systems, Stud. Appl. Math., 101, 197-210 (1998).

[10] R. Grimshaw, L. A. Ostrovsky, V. I. Shrira, \& Y. A. Stepanyants, Long nonlinear surface and internal gravity waves in a rotating ocean, Surveys in Geophysics, 19, n. 4, 289-338 (1998).

[11] R. Grimshaw, E. N. Pelinovsky, \& T. G. Talipova, Damping of large-amplitude solitary waves, Wave Motion, 37, 351-364 (2003).

[12] R. Grimshaw, \& K. Helfrich, Long-time solutions of the Ostrovsky equation, Stud. Appl. Math., 121, 71-88 (2008).

[13] R. Grimshaw, \& K. Helfrich, The effect of rotation on internal solitary waves, IMA J. Appl. Math., 77, 326-339 (2012).

[14] R. Grimshaw, Y. Stepanyants, \& A. Alias, Formation of wave packets in the Ostrovsky equation for both normal and anomalous dispersion, Proc. Roy. Soc. A, 472, 20150416, 20 pp. (2016).

[15] V. I. Karpman, Nonlinear Waves in Dispersive Media (Nauka, Moscow, 1973; Engl. transl.: Pergamon Press, Oxford, 1975).

[16] D. J. Kaup \& B. A. Malomed, Embedded solitons in Lagrangian and semi-Lagrangian systems, Physica D, 184, 153-161 (2003).

[17] Yu. S. Kivshar \& G. Agrawal, Optical Solitons: From Fibers to Photonic Crystals (Academic Press, Amsterdam et al., 2003).

[18] M. J. Lighthill, Contribution to the theory of waves in non-linear dispersive systems, J. Inst. Math. Appl., 1, 269-306 (1965).

[19] B. A. Malomed, Soliton Management in Periodic Systems (Springer, New York, 2006).

[20] J. W. Miles, On the Korteweg-de Vries equation for a gradually varying channel, J. Fluid Mech., 91, 181-190 (1979).

[21] S. P. Nikitenkova, N. Singh \& Y. A. Stepanyants, Modulational stability of weakly nonlinear wave-trains in media with small- and large-scale dispersions, Chaos, 25, 123113, 9 pp. (2015).

[22] C. J. Knickerbocker \& A. C. Newell, Shelves and the Korteweg-de Vries equation, J. Fluid Mech., 98, 803-818 (1980).

[23] L. A. Ostrovsky, Nonlinear internal waves in a rotating ocean, Oceanology, 18, 119-125 (1978).

[24] L. A. Ostrovsky, Asymptotic Perturbation Theory of Waves (Imperial College Press, 2015).

[25] L. A. Ostrovsky, Yu. A. Stepanyants \& L. Sh. Tsimring, Radiation instability in a stratified shear flow, Int. J. 
Non-Lin. Mech., 19 (2), 151-161 (1984).

[26] E. Ott \& R. N. Sudan, Nonlinear theory of ion acoustic waves with Landau damping, Phys. Fluids, 12, 23882394 (1969).

[27] E. Ott \& R. N. Sudan, Damping of solitary saves, Phys. Fluids, 13, 1432-1434 (1970).

[28] V. I. Petviashvili \& O. V. Pokhotelov, Solitary Waves in Plasmas and in the Atmosphere (Moscow, Energoatomizdat, 1989. Engl. transl.: Gordon and Breach, Philadelphia, 1992).

[29] D. E. Pelinovsky \& Y. A. Stepanyants, Convergence of Petviashvili’s iteration method for numerical approximation of stationary solutions of nonlinear wave equations, SIAM J. Numerical Analysis, 42, 1110-1127 (2004).

[30] A. J. Whitfield \& E. R. Johnson, Rotation-induced nonlinear wavepackets in internal waves, Phys. Fluids, 26, 056606 (2014).

[31] A. J. Whitfield \& E. R. Johnson, Wave-packet formation at the zero-dispersion point in the Gardner-Ostrovsky equation, Phys. Rev. E, 91, 051201(R) (2015).

[32] G.B. Whitham, Linear and Nonlinear Waves, J. Wiley and Sons, New York (1974).

[33] N. J. Zabusky \& M. D. Kruskal, Interaction of "solitons" in a collisionless plasma and the recurrence of initial states, Phys. Rev. Lett., 15, 240-243 (1965).

[34] V. E. Zakharov \& L. A. Ostrovsky, Modulation instability: The beginning, Physica D, 238, 540-548 (2009).

[35] Bed roughness and friction factors in estuaries, http://www.marinespecies.org/introduced/wiki/Bed_ roughness_and_friction_factors_in_estuaries 